\title{
APPENDIX B: GLOSSARY OF TERMS
}

(a,b) Nuclear reaction: a target is hit by particle a and particle $b$ is emitted.

\section{AMS Accelerator Mass Spectrometry}

Antiquity The period of human history and prehistory prior to the Middle Ages ( $6^{\text {th }}-15^{\text {th }}$ centuries).

Anti-neutrino A very light, neutral anti-particle emitted in $\boldsymbol{\beta}$-decay.

Alpha (a) particles A heavy, positive, doubly charged particle emitted from some unstable heavy nuclei.

Archaeometallurgy The study of human use of metals in antiquity.

Beta $(\boldsymbol{\beta})$ particles A light, singly charged particle, emitted in $\beta^{-}$-decay. The negatively charged $\beta^{-}$particle has the same properties as an electron. Its $\beta^{+}$anti-particle is positively charged and is emitted in $\beta^{+}$-decays.

Bomb peak A peak in ${ }^{14} \mathrm{C}$ concentration which occurs in biological cells created after the 1950s. It is due to the effects of atmospheric nuclear tests and is relevant to the interpretation of radiocarbon dating measurements.

${ }^{14} \mathrm{C} \quad \beta$-unstable Carbon isotope with 6 protons and 8 neutrons.

Charged particles Particles such as protons and electrons which carry electric charge.

CT Computed tomography: a 2D x-ray or $\mathrm{Y}$-ray image constructed from multiple 1D images taken at different angles.

Concentration The relative abundance of a constituent part or element. Concentration is often presented in units of atomic percent (at.\%).

CPMG sequence Car-Purcell-Meiboom-Gill sequence of radio-frequency pulses commonly used in NMR imaging.

Cross section A measure of the probability, or likelihood, of a reaction taking place

Cultural heritage Artefacts which represent aspects of history of human culture, civilisation and society.

Curation The preservation, cataloguing and safeguarding of historical artefacts.

Deuterium A heavy Hydrogen atom whose atomic nucleus contains one proton plus one neutron.
Dosimetry The measurement of exposure to ionising radiation.

DNA DeoxyriboNucleic Acid. DNA is the building block of biological cells, encoding genetic information.

EDX Energy Dispersive X-ray spectroscopy is an electron-induced $X$-ray technique used for elemental analysis, often used in conjunction with electon microscopy.

Electrons Light, negatively charged particles which are part of each atom, orbiting its positively charged nucleus.

Element A substance which cannot be broken into simpler substances by chemical means. Each element has unique physical and chemical properties and its own place in the periodic table of the elements. An atom is the smallest unit of each element.

Elemental analysis An analysis of which elements which make up an object.

ELI-NP Extreme Light Infrastructure-Nuclear Physics: a very high intensity laser and $\mathbf{y}$-beam facility for Nuclear Physics studies, Bucharest-Magurele, Romania.

ERDA Elastic Recoil Detection Analysis.

$\mathbf{e V}, \mathbf{k e V}, \mathbf{M e V}$ The eV is a unit of energy, specifically the energy an electron acquires when it passes a potential difference of $1 \mathrm{~V}$; keV (1000s of eV) are typical energies of X-rays; $\mathrm{MeV}(1,000,000$ s of $\mathrm{eV})$ are typical energies of $\mathrm{Y}$-rays.

External beam A beam of particles outside a vacuum chamber, often emerging through a thin window.

Y-rays Very high energy electromagnetic radiation in the MeV energy range, produced by high energy bremsstrahlung or transitions between nuclear energy levels.

Ge(Li) Lithium-drifted Germanium solid state detector, used to measure $\gamma$-ray energies.

Half-life The characteristic time taken for the intensity of a radioactive substance to reduce by a factor of two.

Heavy ions Charged atoms from heavy elements

HIERDA Elastic Recoil Detection Analysis using a heavy ion beam.

Homogeneous Having the same value of some quantity

HPGe Hyper-pure Germanium detector, used to measure Y-ray energies. 
IAEA International Atomic Energy Agency

Ion Beams A beam, or flow, of flux of positively, or negatively, charged atoms

IBA Ion Beam Analysis.

IBIL Ion Beam Induced Luminescence.

INAA Instrumental Neutron Activation Analysis.

INFN Italian Nuclear Physics Research Agency: Istituto Nazionale di Fisica Nucleare

Inhomogeneous Having non-constant values of some quantity

In-situ In its customary position, not in a laboratory.

Ionising radiation Radiation which causes ionisation of atoms. Ionising radiation includes ion beams, a-particles, $\beta$-particles, $\gamma$-rays and $X$-rays. Neutron radiation does not directly cause ionisation, but can also lead to the break-up of atoms.

Irradiation Subjecting an object to a dose of radiation.

ISIS Research laboratory near Oxford, UK, with spallation neutron beam facilities.

Isotope An atom with a particular number of neutrons in its nucleus.

LABEC Laboratorio di Tecniche Nucleari per i Beni Culturali, in Florence, Italy.

Luminescence Emission of light from energy stored in excited atomic states.

OSL Optically-stimulated luminescence.

NAA Neutron Activation Analysis.

NDP Neutron Depth Profiling.

Neutrons Heavy neutral particles, found as constiuents of atomic nuclei.

NMR Nuclear Magnetic Resonance.

NMR-MOUSE Nuclear Magnetic Resonance-Mobile Universal Surface Explorer: a small portable NMR measurement system capable of measuring depth profiles.

NRA Nuclear Reaction Analysis.

NRF Nuclear Resonance Fluorescence.

$\boldsymbol{\mu}$-IBIL Micro-beam Ion Beam Induced Luminescence.

$\mathbf{\mu - X R F \quad M i c r o - b e a m ~ X - R a y ~ F l u o r e s c e n c e . ~}$

PGAA or PGNAA Prompt Gamma Activation Analysis.

PIGE Particle-Induced $\gamma$-ray Emission.

PIGME Particle-Induced $\gamma$-ray Emission.

PIXE Particle-Induced X-ray Emission.
PMP Proton Micro Probe.

Protons Heavy positively charged particles, found as constiuents of all atomic nuclei.

Provenance The source or origin of an object or artefact.

Radiocarbon dating Dating of historical objects containing carbon by measuring their ${ }^{14} \mathrm{C}$ concentration.

Raman Spectroscopy Optical technique used to identify molecules.

RBS Rutherford Back-Scattering.

RBS/C RBS-Channelling spectrometry.

REE Rare Earth Element. One of 17 heavy elements which have a low natural abundance. Rare Earth Elements include the complete Lanthanide series of elements

Reactor A vessel in which a controlled nuclear reaction takes place. Reactors are commonly used as intense sources of neutron beams.

RNAA Radiochemical separation of a sample undergoing Neutron Activation Analysis.

Relaxation time The characteristic time taken for magnetic polarisation to decay in NMR studies.

RNRA Resonant Nuclear Reaction Analysis.

SANS Small Angle Neutron Scattering.

SEM-EDX Scanning Electron Microscopy coupled with Energy Dispersive X-ray spectroscopy.

SDD Silicon Drift Detector: a semiconductor detector commonly used to detect $\mathrm{X}$-rays.

SIMP Scanning Ion Micro Probe.

SINQ The Swiss Spallation Neutron Source at the Paul Scherrer Institute, Villigen, Switzertland.

$\mathrm{Si}(\mathrm{Li}) \quad$ Lithium-drifted Silicon solid state detector, used to measure $\mathrm{X}$-ray energies

TL Thermo-Luminescence.

Trace element Elements which occur as a low-concentration constituent or impurity in an object.

TOF Time-of-Flight: a technique used to measure particle energies by the time they take to cover a known distance.

Triptych A three-panel painting or icon.

TOF-ND Time-of-flight neutron diffraction.

X-rays High energy electromagnetic radiation in the keV energy range, produced by electron bremsstrahlung or transitions between atomic energy levels.

XRF X-Ray Fluorescence. 